\title{
Tratamiento quirúrgico de acné queloide nucal
}

\author{
Acne keloidalis nuchae surgical treatment
}

\author{
Dra. Claudia Gutiérrez-Gómez,* Dra. Brenda Valdez-Díaz
}

Palabras clave: Acné queloide nucal, foliculitis queloide, acné queloide.

Keywords: Acne keloidalis nuchae, folliculitis keloidalis, acne keloidalis.

* Cirujana Plástica y Reconstructiva, Hospital General «Dr. Manuel Gea González». Laboratorio de Biotecnología del Centro Nacional de Quemados del Instituto Nacional de Rehabilitación «Luis Guillermo Ibarra Ibarra».

‡ Residente de cirugía plástica y reconstructiva, Instituto Mexicano del Seguro Social.

Ciudad de México.

Recibido:

08 agosto 2020

Aceptado:

14 septiembre 2020

\section{RESUMEN}

El acné queloide nucal es un proceso inflamatorio crónico del folículo piloso de la región occipital que conduce a la formación de placas fibrosas, pápulas y alopecia. En casos severos, el acné queloide nucal conlleva considerables efectos psicológicos y afecta la calidad de vida. El manejo médico tradicional se enfoca en la prevención, el uso de antibiótico tópico u oral y esteroides intralesionales para disminuir la inflamación y la infección secundaria. Desafortunadamente el tratamiento médico requiere meses para alcanzar resultados poco satisfactorios y son frecuentes las recurrencias. El manejo quirúrgico de las lesiones es invasivo, requiere mayor tiempo para la recuperación. La crioterapia, el dióxido de carbono y la ablación con láser son alternativas para el tratamiento de la foliculitis queloide, con resultados limitados. El objetivo de este trabajo es analizar la técnica utilizada y el resultado en un caso de acné queloide nucal tratado con colocación de expansor tisular. Describimos el caso de un paciente de 33 años de edad con diagnóstico histopatológico de foliculitis queloide tratado con resección amplia y avance de colgajo con previa colocación de expansor. Después de dos años de seguimiento no observamos recurrencia y se obtuvo un buen resultado estético. La expansión tisular es un recurso útil para el manejo definitivo de lesiones extensas y refractarias a tratamiento. Algunos autores han reportado el uso de radioterapia adyuvante postresección, en el presente caso no se utilizó, no presentó recidiva en un seguimiento a dos años. Concluimos que se han utilizado numerosos tratamientos para el acné queloide nucal, desde antibióticos tópicos hasta resección quirúrgica de las lesiones fibróticas. Es de suma importancia individualizar cada caso para realizar el tratamiento más adecuado según el contexto clínico.

\section{ABSTRACT}

Acne keloidalis nuchae is a chronic inflammatory condition of the hair follicle on the occiput that leads to fibrotic plaques, papules and alopecia. In severe cases, acne keloidalis nuchae leads to considerable psychological effects and affects quality of life. Traditional medical management focuses on prevention, the use of oral ant topic antibiotics, and intralesional steroids in order to decrease inflammation and secondary infections. Unfortunately, therapy may require months of treatment to achieve results which are not satisfactory and recurrences are common. A surgical handling treat lesion is invasive and requires more time to recover. Cryotherapy, carbon dioxide and laser ablation therapies are alternative treatments of folliculitis keloidalis with limited results. The objective of this work is to analyze the technique used and the results of a case of acne keloidalis nuchae treated with tissue expansion. We described a case of 33 year old patient with histopathological diagnosis of folliculitis keloidalis treated with wide resection and flap advance with previous placement of expander. After two years follow-up we did not observe recurrence, and a good cosmetic result was achieved. Tissue expansion is a useful resource for definitive management of extended lesions and refractory to medical treatment. Some authors have reported the use of adjuvant radiotherapy postop, which was not used in this case, and there was no recurrence in a two year follow-up. We concluded that many treatments have been performed for acne keloidalis nuchae from topical antibiotics to surgical excision of fibrotic plaques. It is very important to consider each case to carry out the adequate treatment according to the clinical context.

\section{INTRODUCCIÓN}

T a foliculitis queloide (FQ) es un proceso $ـ$ inflamatorio crónico del folículo piloso de la región occipital que conduce a la formación de placas fibrosas, pápulas y alopecia. En casos severos la FQ conlleva considerables efectos psicológicos y afecta la calidad de vida.

Es más frecuente en pacientes jóvenes varones de raza negra, aunque puede encontrarse

Citar como: Gutiérrez-Gómez C, Valdez-Díaz B. Tratamiento quirúrgico de acné queloide nucal. Cir Plast. 2020; 30 (3): 156-159. https://dx.doi.org/10.35366/98340 
en hispanos, asiáticos, blancos y en mujeres. También los fototipos cutáneos de Fitzpatrick $\mathrm{IV}, \mathrm{V}$ y $\mathrm{VI}$, y algunos cortes de cabello parecen ser factores precipitantes para esta enfermedad.

La prevalencia reportada es de $13.7 \%$ en hombres de raza negra. Los estudios reportan una incidencia entre 0.45 y $9 \%$ en pacientes dermatológicos, la mayoría en raza negra con cabello rizado y parece aumentar con el tiempo. Se han propuesto distintos mecanismos, las dos teorías predominantes sugieren que la lesión de la piel y la existencia de reacciones inmunes son las causas principales. El aumento en la densidad de mastocitos contribuye al prurito y a la manipulación mecánica de la región. ${ }^{1-4}$

La forma indicada para tratar a los pacientes con FQ consiste principalmente en reducir los traumatismos, como el roce del cuello con la camisa, cortarse el cabello muy corto o afeitarlo y evitar el uso de agentes químicos agresivos para el cabello. La farmacoterapia de primera línea indica el uso de esteroides potentes de uso tópico. Los retinoides tópicos en combinaciones con los corticosteroides ayudan a disminuir las lesiones. Cuando las lesiones se infectan de manera secundaria está indicado el uso de antibiótico oral o tópico. Los corticoides intralesionales inyectados son efectivos para lesiones extensas y deben ser considerados para casos resistentes a la monoterapia tópica. Los casos avanzados de FQ pueden requerir manejo quirúrgico; otras opciones terapéuticas incluyen las de mínima invasión, como la crioterapia, dióxido de carbono, ablación con

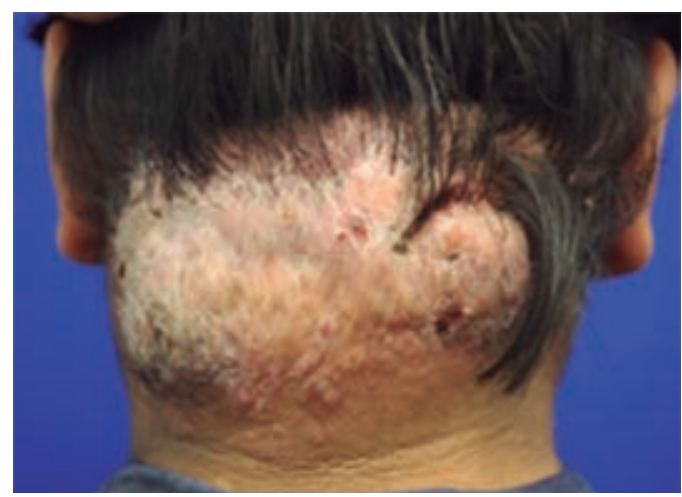

Figura 1: Hombre de 33 años de edad con lesión en región occipital de $11 \times 8 \mathrm{~cm}$. Vista preoperatoria.

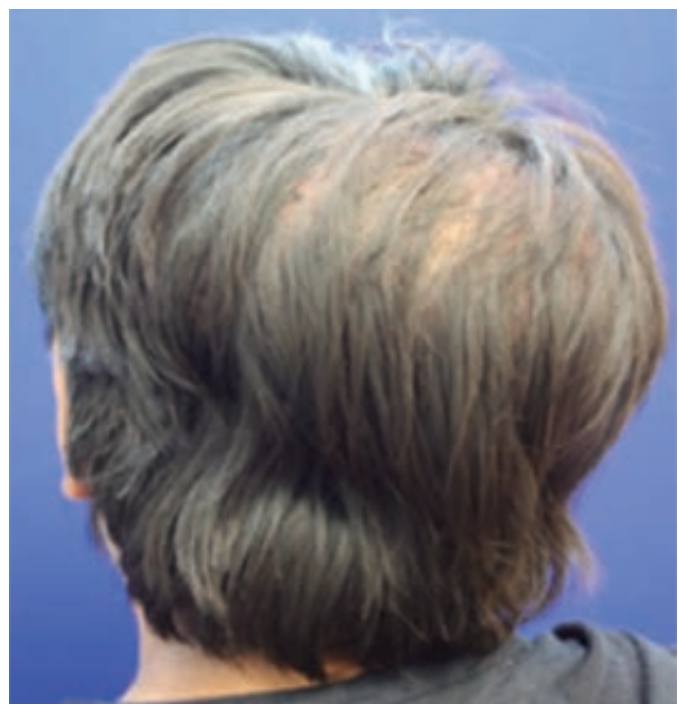

Figura 2: Una vez colocado el expansor tisular en región parietooccipital y terminada la expansión con un volumen de $800 \mathrm{~mL}$.

láser o depilación del vello con láser diodo con resultados limitados. ${ }^{2,4}$

\section{CASO CLÍNICO}

Hombre de 33 años de edad con antecedente de presentar, con un año de evolución, múltiples pápulas eritematosas dolorosas y pruriginosas en la región occipital, que ulteriormente tuvieron salida de material purulento y mal olor. Estas lesiones se extendieron alcanzando un tamaño aproximado de $11 \times$ $8 \mathrm{~cm}$, con tratamiento previo con tetraciclina y Retin-A.

Un año después, el paciente presentó prurito, dolor, salida de material purulento y trayectos fistulosos. Se inició manejo con trimetoprima con sulfametoxazol e ibuprofeno y se realizó biopsia con reporte diagnóstico de foliculitis queloide nucal (Figura 1).

Se colocó un expansor tisular liso rectangular de $15 \times 7.5 \mathrm{~cm}$, de $750 \mathrm{~mL}$ de volumen (Silimed 810-720-3), con válvula remota en la región frontoparietal y llenado transoperatorio de $40 \mathrm{~mL}$, por medio de incisión de $4 \mathrm{~cm}$ perpendicular al eje del expansor, $10 \mathrm{~cm}$ arriba del borde superior de la lesión, a nivel de la región parietooccipital. A los 30 días de la colocación del expansor se iniciaron infiltraciones sema- 


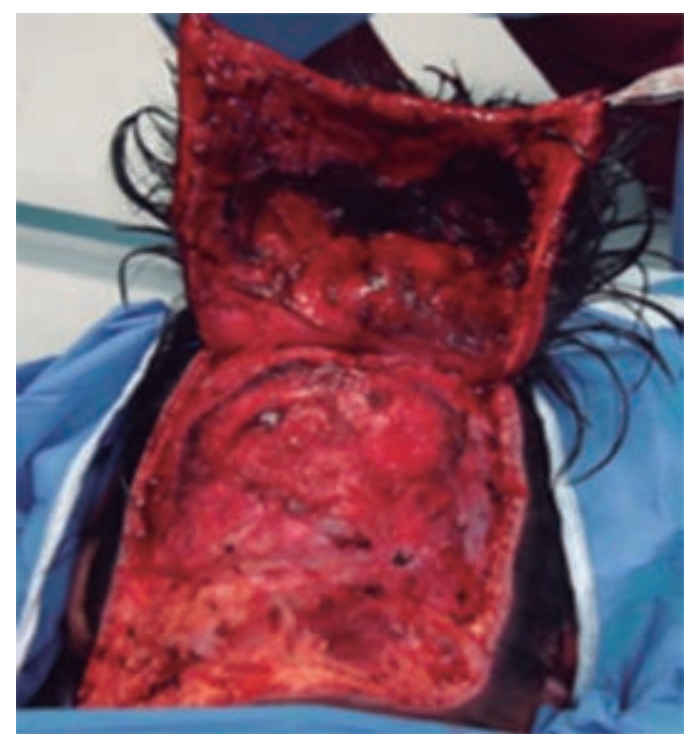

Figura 3: Vista transoperatoria una vez retirado el expansor y resecada la lesión. Colgajo rectangular de avance de pedículo superior.

nales de $75 \mathrm{~mL}$ hasta alcanzar un volumen de $800 \mathrm{~mL}$ (Figura 2).

Tres meses después de terminada la expansión se retiró el expansor, se realizó un colgajo cuadrangular de base superior y resección completa de la zona afectada (Figura 3). En un seguimiento de dos años, no observamos recurrencia del acné queloide nucal y se obtuvo un buen resultado estético (Figura 4).

\section{DISCUSIÓN}

Lo ideal es el tratamiento quirúrgico para los casos severos de FQ que cursan con una masa tumoral. Entre los tratamientos quirúrgicos propuestos se encuentran la resección más injerto, resección con cierre primario en los casos que esto lo permite, resección con cicatrización espontánea, resecciones seriadas con cierre directo y, en casos extremos, con expansión tisular. ${ }^{5-8}$ La colocación de injertos tiene el inconveniente de requerir de un área donadora, con un pobre aspecto estético por la pigmentación de los injertos. Por otro lado, la cicatrización por segunda intención también tendrá un resultado poco estético en cuanto a cicatrización y retracción. La expansión tisular es un recurso útil para el manejo definitivo de lesiones extensas y refractarias a tratamiento, como se presentó en este paciente. El colgajo de avance de piel cabelluda y la resección de la cicatriz queloide nucal no hubieran sido posibles sin la colocación previa del expansor tisular. Otro tipo de manejo en este caso hubiera tenido escasos resultados estéticos, ocasionando alopecia occipital. Por último, tras dos años de seguimiento, no observamos recurrencia de foliculitis queloide ni signos del fenómeno «stretch-back» después de la colocación del expansor. Si bien, en el caso reportado por Pestalardo y colaboradores en el que aplicaron radioterapia después del avance de colgajo con expansión tisular, consideramos que si se evitan los factores locales y se hace un cierre sin tensión, no habrá necesariamente una recidiva, como el caso que presentamos a dos años de evolución.

\section{CONCLUSIÓN}

Se han utilizado múltiples tratamientos para el acné queloide nucal, desde antibióticos tópicos hasta resección quirúrgica con aplicación de injertos, o cierre por segunda intención. El manejo médico tradicional requiere aplicaciones diarias durante meses y puede presentar recurrencia tras su suspensión. Los tratamientos alternativos pueden tener pocos resultados

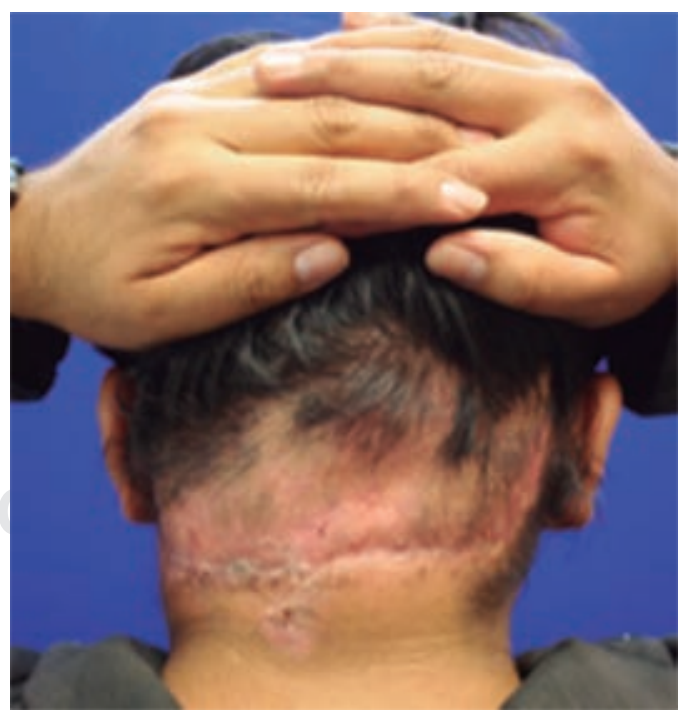

Figura 4: Vista postoperatoria a seis meses con resultado del avance del colgajo. 
estéticos así como recidivas. El manejo quirúrgico se reserva para casos refractarios y lesiones extensas. De igual forma, se ha reportado el uso de radioterapia en casos de difícil control, pero se deben considerar los efectos adversos de dicho manejo; no obstante, cabe destacar la importancia de individualizar cada caso para realizar el tratamiento más adecuado según el contexto clínico.

\section{REFERENCIAS}

1. Coley MK, Kelly AP, Alexis AF. Pseudofolliculitis barbae and acne keloidalis nuchae. In: Alexis AF, Barbosa VH, Ed. Skin of color: a practical guide to dermatologic diagnosis and treatment. New York: Springer; 2013, pp. 123-137.

2. Maranda EL, Simmons BJ, Nguyen AH et al. Treatment of acne keloidalis nuchae: a systematic review of the literature. Dermatol Ther 2016; 6: 363-378.

3. Reja M, Silverberg NB. Acne keloidalis nuchae. In: Silverber NB et al (Ed). Pediatric skin of color. New York: Springer; 2015, pp. 141-145.

4. Ogunbiyi A, Adedokun B. Perceived etiological factors o folliculitis keloidalis nuchae (acne keloidalis) and treatment options among Nigerian men. Br J Dermatol 2015; 173 (2): 22-25.

5. Gloster Jr HM. The surgical management of extensive cases of acne keloidalis nuchae. Arch Dermatol 2000; 136: 1376-1379.

6. Bajaj V, Langtry JAAA. Surgical excision of acne keloidalis nuchae with secondary intention healing. Clin Exp Dermatol 2007; 33: 53-55.

7. Dinehart SM, Herzberg AJ, Kerns B J et al. Acne keloidalis: a review. J Dermatol Surg Oncol 1989; 15: 642-647.

8. Prestalardo CM, Cordero A, Mato J, Bestue M, Martinho A. Acne keloidalis nuchae tissue expansion treatment. Dermatol Surg 1995; 21: 723-724.

Correspondencia:

Dra. Claudia Gutiérrez Gómez

Puente de Piedra Núm. 150 T2 C 420

Col. Toriello Guerra, 14050

Alcaldía Tlalpan, Ciudad de México.

Correo electrónico: dra.claugg8@gmail.com

Conflicto de intereses: Los autores declaran no tener conflicto de intereses. 